\title{
SCIENTIFIC REPORTS

\section{OPEN First demonstration of protective effects of purified mushroom polysaccharide-peptides against fatty liver injury and the mechanisms involved}

Received: 15 January 2019

Accepted: 31 August 2019

Published online: 23 September 2019

\begin{abstract}
Shuang Zhao ${ }^{1,2}$, Shuman Zhang ${ }^{1}$, Weiwei Zhang ${ }^{3}$, Yi Gao ${ }^{4}$, Chengbo Rong ${ }^{1}$, Hexiang Wang ${ }^{5}$, Yu Liu' ${ }^{1}$, Jack Ho Wong ${ }^{6}$ \& Tzibun $\mathrm{Ng}^{6}$

Fatty liver (FLD) disease is a consequence of metabolic syndrome, which is a health problem worldwide with a phenomenal rise in prevalence. In this study, two hepatoprotective polysaccharide-peptides were extracted from the mushroom Auricularia polytricha followed by chromatographic fractionation of the extract on the ion exchanger DEAE-cellulose and gel filtration on Sephadex-200 to yield two purified fractions: APPI and APPII. The monosaccharide compositions, FT-IR, N-terminal sequences, internal peptide sequences and molecular weights of the two fractions were determined. Furthermore, their hepatoprotective effect on human hepatoma HepG2 cells in vitro and in an animal model of fatty liver disease was evidenced by the findings that APPI and APPII diminished lipid deposit in cells, blood and the liver, increased cellular antioxidant activity and viability, and protected the liver against injury. The mechanistic study revealed that APPI and APPII activated the adiponectin pathway, up-regulated expression of genes controlling free fatty acid (FFA) oxidation, such as AMPK, CPTI, ACOX1 and PPAR $\alpha$ genes, enhanced lipid metabolism, preserved hepatic function, promoted the antioxidant defense system and reduced lipid peroxidation. Hence the bioactive compounds of $A$. polytricha could serve as therapeutic agents in the food and pharmaceutical industries.
\end{abstract}

The liver is an important organ in charge of the metabolism of lipids, glucose, proteins, alcohol, drugs, and chemicals ${ }^{1}$. Accumulating data suggest that excessive intake of alcohol and fat induces improper triglyceride metabolism in the hepatocytes ${ }^{2}$. When synthesis proceeds faster than anabolism, triglycerides accumulate in the liver. The excessive lipid in the hepatocytes, exceeding $5-10 \%$ of the liver weight, is the main pathogenetic factor of fatty liver disease (FLD) ${ }^{3,4}$. The multiplicity of etiologic factors of FLD, include genetic, dietary factors, insulin resistance, and adipokines 5 . FLD is a metabolic syndrome, which has become a vital health issue with a phenomenal escalation in prevalence. Fatty liver disease comprises alcoholic fatty liver disease and nonalcoholic fatty liver disease (NAFLD). Alcohol-related liver disease and NAFLD are common chronic hepatic derangements ${ }^{6}$. The disease affects grown-ups and children alike and is getting more and more rampant in industrialized as well as developing countries. Cirrhosis associated with NAFLD is likely to be a priority for hepatic transplantation in the foreseeable future. The incidence of liver cancer associated with NAFLD shows a rising trend ${ }^{7}$. Cardiometabolic

${ }^{1}$ Institute of Plant and Environment Protection, Beijing Academy of Agriculture and Forestry Sciences, Beijing, 100097, China. ${ }^{2}$ Beijing Key Laboratory of Fruits and Vegetable Storage and Processing, Key Laboratory of Vegetable Postharvest Processing, Ministry of Agriculture, Beijing, 100097, China. IInstitute of Medicinal Plant Development, Chinese Academy of Medical Sciences \& Peking Union Medical College, Beijing, 100193, China. ${ }^{4}$ Beijing Xicheng District Health Care Center for Mothers and Children, Beijing, 100053, China. ${ }^{5}$ State Key Laboratory for Agrobiotechnology and Department of Microbiology, China Agricultural University, Beijing, 100193, China. ${ }^{6}$ School of Biomedical Sciences, Faculty of Medicine, The Chinese University of Hong Kong, Shatin, New Territories, Hong Kong, China. Shuang Zhao, Shuman Zhang and Weiwei Zhang contributed equally. Correspondence and requests for materials should be addressed to H.W. (email: hxwang@cau.edu.cn) or J.H.W. (email: jack1993@yahoo.com) orT.N. (email: b021770@mailserv.cuhk.edu.hk) 
disorders, dyslipidemia in particular, are often associated with pediatric NAFLD ${ }^{8}$. Approximately one-third of the Americans have NAFLD with consequent heavy economic and societal burdens ${ }^{9}$. Treatments for NAFLD are found to be not optimal. Some of the drugs used have side effects ${ }^{10}$. Hence, it would be highly desirable to devise a management plan to ascertain efficacious natural products for human populations at high risk of developing fatty liver disease in order to forestall or retard the exacerbation of hepatic damage at an early stage. To combat this disease, natural products may have value ${ }^{11}$.

Currently, growing attention has been drawn to the exploitation of biomedicines owing to their minimal toxicity and therapeutic efficacy. Polysaccharide, a potential agent that meets the requirement, is found in many vegetables, fruits, edible fungi and other microorganisms. Increasing studies furnish supporting evidence demonstrating that polysaccharides display a diversity of biological activities, encompassing antioxidant ${ }^{12}$, anti-tumor ${ }^{13}$, antidiabetic $^{14,15}$, renoprotective $\mathrm{e}^{16,17}$, immunomodulatory ${ }^{18}$ and hepatoprotective ${ }^{19,20}$ activities. Polysaccharides isolated from mushrooms have been extensively investigated in the medicinal arena owing to the ready availability of fermentation technology $y^{21-23}$.

Mushrooms exert a myriad of health promoting actions. Thus mushrooms have captured the attention of many researchers. Two important activities are hepatoprotective and antihyperlipidemic activities ${ }^{24-26}$. Polysaccharides from mushrooms exhibit diverse activities: those with hepatoprotective activities have been isolated from Agaricus bisporus, Coprinus comatus, Hypsizigus marmoreus, Oudemansiella radicata, Pholiota dinghuensis, Pleurotus eryngii, Pholiota nameko, Pleurotus djamor, and Russula vinosa ${ }^{27-37}$. Polysaccharides with antihyperlipidemic activity have been isolated from Pholiota nameko and Termitomyces albuminosus ${ }^{38,39}$.

Auricularia polytricha, alternatively referred to as wood ear or Jew's ear, belonging to Auriculariaceae family, is a culinary-medicinal fungus which manifests a multitude of activities. Modern research indicates that $A$. polytricha is a kind of healthy mushroom with a high carbohydrate content (about $630 \mathrm{~g} / \mathrm{kg}$ in dried fruiting bodies $)^{40}$. The polysaccharides from the fruiting bodies have multiple pharmacological functions with potential for clinical application and are devoid of toxicity and significant side effects. After oral administration a soluble polysaccharide from $A$. polytricha exhibited antihypercholesterolemic activity in rats ${ }^{41}$. An ethanolic extract of mycelial culture exhibited antioxidant and tyrosinase inhibitory activities ${ }^{42}$. An aqueous extract of fruiting bodies demonstrated hepatoprotective effect against paracetamol in rats ${ }^{43}$. An aqueous extract was devoid of cytotoxicity toward normal kidney NRK-52E cells ${ }^{44}$ but displayed antiproliferative effect on COLO-205 colon cancer cells ${ }^{45}$. Polysaccharides with anticancer activity in $\mathrm{S} 180$ sarcoma bearing mice ${ }^{46}$, antimutagenic activity against the alkylating agent cyclophosphamide ${ }^{47}$, and antiproliferative, cell cycle arresting and apoptotic activities toward A549 human lung cancer cells ${ }^{48}$ have been isolated from A. polytricha. A hot water extract manifested hypoglycemic effects $^{49}$. Hot water extracts inhibited platelet aggregation with a mechanism independent of cyclic AMP ${ }^{50,51}$. Thermostable constituents suppressed activity of beta secretase which liberates toxic $\beta$-amyloid peptide in the brain and protects against neurodegenerative diseases such as Alzheimer's disease ${ }^{52}$. Polysaccharides with different activities have been reported from $A$. polytrich $a^{41,46-48}$. Three anticancer polysaccharides AAPS-1, AAPS-2, and AAPS-3 with molecular weights of 162,259 , and $483 \mathrm{kDa}^{46}$ and an antimutagenic $930 \mathrm{kDa}$ salt-soluble polysaccharide ${ }^{47}$ from $A$. polytricha have been reported.

Our previous studies indicated that polysaccharide prepared from A. polytricha effectively lowered the levels of total cholesterol, LDL-cholesterol and triglycerides ${ }^{41}$ in the blood circulation, indicating that the polysaccharide could regulate the metabolism of lipids, and is exploitable for alleviation of FLD and liver injury. Nevertheless, to date, no detailed studies have been carried out on the characterization, hepatoprotective effect and mechanism of polysaccharides or polysaccharide-peptides from A. polytricha. Thus we commenced this investigation on purification and characterization of bioactive polysaccharide-peptides, exploring their hepatoprotective effect and mechanism with the intent to ascertain novel bioactive constituents utilizable in the food and pharmaceutical industries.

\section{Results}

Extraction and purification of APPI and APPII. The crude polysaccharide-peptide from A. polytricha fruiting bodies was firstly resolved on DEAE-cellulose. According to the charge difference, two peaks, D1 and D2, eluted with 0 and $0.2 \mathrm{~mol} / \mathrm{L} \mathrm{NaCl}$ solution respectively, were detected by using the phenol-sulfuric acid method (see Supplementary Fig. S1a). The two fractions were then subjected to concentration, dialysis and gel-filtration chromatography on Superdex-200. As a result, both D1 and D2 generated only a single peak, named A. polytricha polysaccharide-peptide I (APPI) and A. polytricha polysaccharide-peptide II (APPII), respectively (refer to Supplementary Fig. S1b,c). The yields of APPI and APPII were approximately $16.31 \%$ and $49.46 \%$ (w/w) based on the weights of dried crude polysaccharide-peptide. High performance gel permeation chromatography (HPGPC) analysis disclosed that the weight-average $(\mathrm{Mw})$, number-average $(\mathrm{Mn})$, and $\mathrm{z}$ average $(\mathrm{Mz}) \mathrm{molecular}$ weights and polydispersity ratio $(\mathrm{Mw} / \mathrm{Mn})$ of APPI were $9.213 \times 10^{5}, 5.568 \times 10^{5}, 1.057 \times 10^{6}$ and 1.655 , while those of APPII were $6.340 \times 10^{5}, 7.693 \times 10^{4}, 9.547 \times 10^{5}$ and 8.241 , respectively.

Characterization of APPI and APPII. APPI and APPII exhibited the same monosaccharide moieties but different molar ratios of monosaccharides and molecular weights, similar FTIR spectra. APPI and APPII are composed of the same monosaccharide moieties, but at different ratios. Monosaccharide composition analysis revealed mannose and xylose are the major sugars, with glucose and small amounts of arabinose and galactose (see Supplementary Fig. S2a,b). APPI was composed of Ara, Gal, Glc, Xyl and Man, in a molar ratio of 1:4.4:15.4:38.3:46.2, while the corresponding molar ratio for APPII was 1:71.5:99.2:10:5.1.

Fourier transform infrared spectroscopy (FTIR) is an important method to predict the structures of natural macromolecules such as polysaccharide-peptides. To more precisely characterize polysaccharide-peptide fractions, the characteristic absorption of polysaccharides was performed in the region $4000-600 \mathrm{~cm}^{-1}$ by FTIR spectrum. As shown in the FTIR spectrum of APPI (Fig. 1a), the intense broad peak at $3400.42 \mathrm{~cm}^{-1}$ was typical 

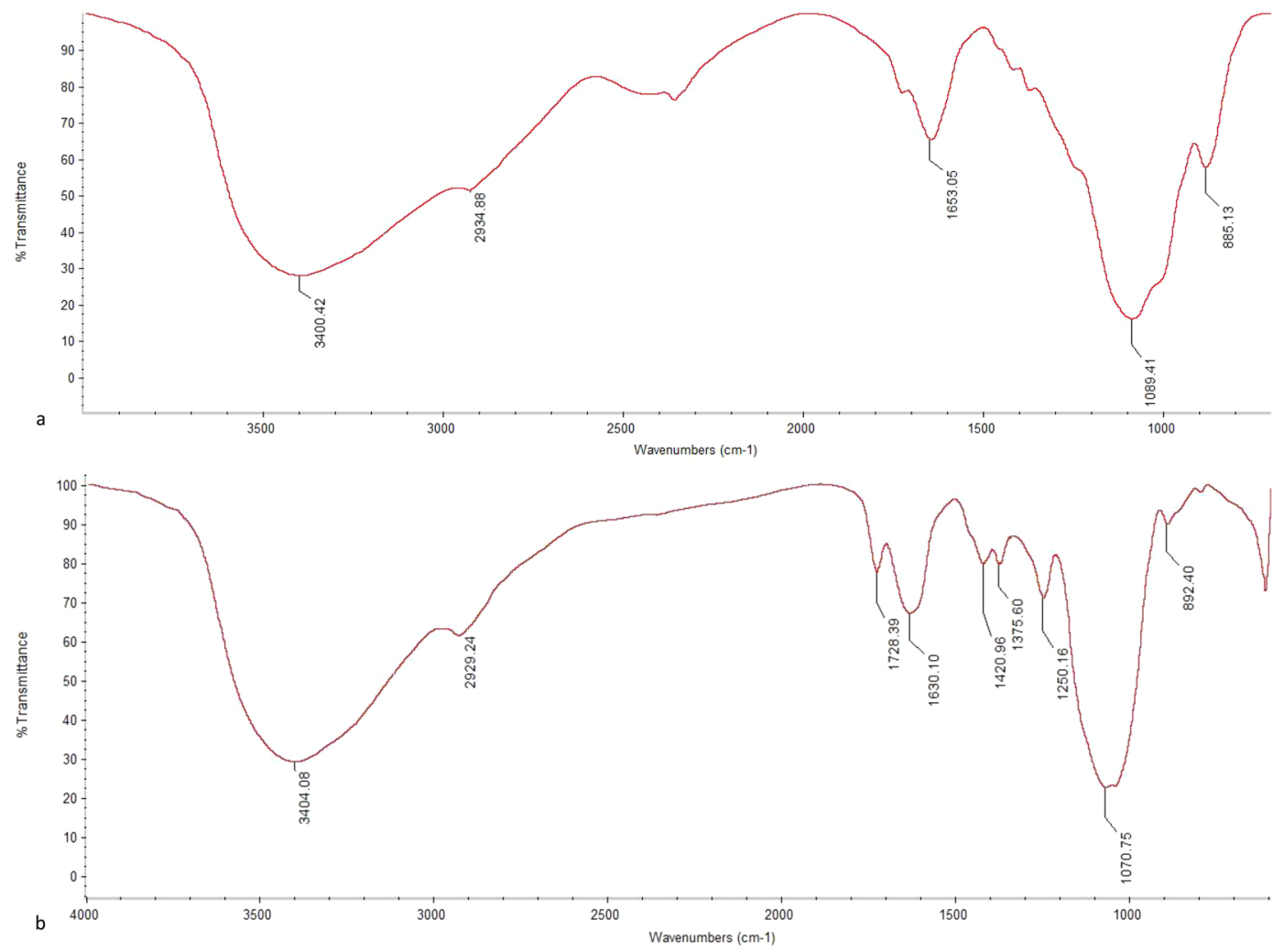

Figure 1. FT-IR spectrum of A. polytricha polysaccharide-peptides. (a) APPI, (b) APPII.

of hydroxyl groups with stretching vibration ${ }^{53}$, and the peak at $2934.88 \mathrm{~cm}^{-1}$ was attributed to C-H stretching vibration ${ }^{54}$. The peak in this region is typical of sugar. The peak at $1653.05 \mathrm{~cm}^{-1}$ suggested the existence of $\mathrm{C}=\mathrm{O}$ bands $s^{55}$. The absorptions between 1000 and $1200 \mathrm{~cm}^{-1}$ were typical absorption peaks of the pyranose ring and hence disclosed the existence of $\mathrm{C}-\mathrm{O}-\mathrm{H}$ side groups and $\mathrm{C}-\mathrm{O}-\mathrm{C}$ glycosidic band vibrations ${ }^{56}$, and the absorption around $880 \mathrm{~cm}^{-1}$ to $900 \mathrm{~cm}^{-1}$ was attributed to the presence of $\alpha$-type glycosidic linkages ${ }^{57}$. The FT-IR spectrum of APPII is shown in Fig. 1b, which was similar to those of APPI (Fig. 1a), but the broad absorption bands of APPII with strong intensities around $1420.96 \mathrm{~cm}^{-1}$ probably reflected deforming vibrations of the C-H bond. Absorption peaks were detected at $1375.60 \mathrm{~cm}^{-1}$ and $1250.16 \mathrm{~cm}^{-1}$, which revealed that the polysaccharide-peptide APPII possessed a carboxyl group and a sulfate radical ${ }^{58}$.

The amino acid sequences of APPI and APPII at the N-terminal were DLYEVVEGEI, and VPSSMVVVVG, respectively. Results from internal amino acid sequence analysis showed that two peptide sequences of APPI, namely VQNVGNGVLLGFHGR and HQTSGDQVTSSTQHSFR, were strikingly similar to mannose-binding lectin from Cordyceps militaris. The peptide sequence GTPSSYIDNLTFPK of APPII manifested considerable homology with immunomodulatory protein from Flammulina velutipes. Another peptide sequence of APPII, ELATGQNGFGYAGSSFHR, demonstrated pronounced resemblance to peptidyl-prolyl cis-trans isomerase from Lentinula edodes.

Protective effects of APPI and APPII in vitro. HepG2 cells of the injury model were exposed to different concentrations of FFA and ethanol, and cell viability was evaluated with the 3-(4,5-dimethylthiazol-2-yl)-2,5-diph enyltetrazolium bromide (MTT) assay. It was found that cells could retain around $60 \%$ viability when $0.5 \mathrm{mmol} / \mathrm{L}$ FFA and $1.8 \%(\mathrm{v} / \mathrm{v})$ ethanol were used to induce hepatic injury. Triglyceride (TG) deposits in the cells were revealed by the Oil-red $\mathrm{O}$ staining method.

The protective effects of APPI and APPII against cell injury induced by pathogenetic factors was assessed by various assays comprising the MTT test, together with assays of intracellular TG, total superoxide dismutase (SOD), and extracellular aspartate transaminase (AST) and alanine aminotransferase (ALT) activities. It was shown that APPI and APPII were not cytotoxic to HepG2 cells in the concentration range of $0-100 \mu \mathrm{g} / \mathrm{mL}$ (Fig. 2a). The cells were exposed to increasing concentrations of APPI or APPII $(30-60 \mu \mathrm{g} / \mathrm{mL})$ for $24 \mathrm{~h}$ after the addition of $0.5 \mathrm{mmol} / \mathrm{L}$ FFA and $1.8 \%(\mathrm{v} / \mathrm{v})$ ethanol. As shown in Fig. 2b, FFA and ethanol treatment brought about significant toxicity to HepG2 cells, whereas treatment with APPI or APPII increased cell viability with the highest ratio of $33.47 \%$ at $40 \mu \mathrm{g} / \mathrm{mL}$ and $23.23 \%$ at $50 \mu \mathrm{g} / \mathrm{mL}$, respectively. The results showed that intracellular TG in APPI and APPII groups was attenuated relative to that in the model group $(\mathrm{P}<0.05)$, as shown in Table 1 , which was also verified through morphological observation aided by the Oil-red O stain (see Supplementary Fig. S3). APPI and APPII elevated the activity of total SOD in HepG2 cells considerably, by $72.72 \%$ and $58.43 \%$, relative to the model group (Table 1). The most conspicuous effect of hepatic injury is the liberation of intracellular enzymes, like aminotransferases, into the circulation, which ensues in an escalation of the activities of these 

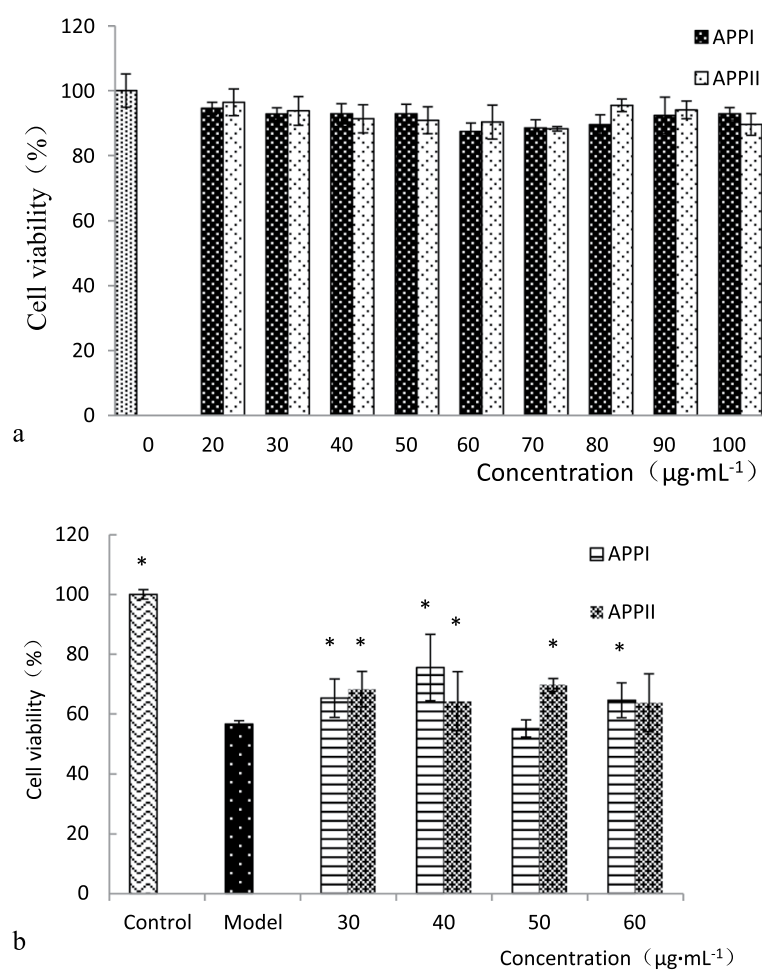

Figure 2. Effects of APPI and APPII in vitro, (a) Cytotoxicity, (b) Hepatoprotective effect. Data are shown as means $\pm \operatorname{SEM}(\mathrm{n}=3) . * \mathrm{P}<0.05$ versus the model group as revealed by ANOVA.

\begin{tabular}{|l|l|l|l|l|}
\hline Groups & TG $(\boldsymbol{\mu m o l} / \mathbf{m g}$ protein) & $\begin{array}{l}\text { T-SOD }(\mathbf{U} / \mathbf{m g} \\
\text { protein })\end{array}$ & ALT (U/L) & AST (U/L) \\
\hline Control & $261.48 \pm 25.19^{*}$ & $24.22 \pm 0.84^{*}$ & $11.34 \pm 0.48^{*}$ & $9.43 \pm 0.92^{*}$ \\
\hline Model & $745.51 \pm 59.22$ & $11.63 \pm 1.24$ & $23.19 \pm 0.76$ & $21.29 \pm 0.58$ \\
\hline APPI & $604.04 \pm 58.07^{*}$ & $20.08 \pm 1.23^{*}$ & $15.24 \pm 1.11^{*}$ & $13.79 \pm 0.43^{*}$ \\
\hline APPII & $472.84 \pm 49.46^{*}$ & $18.41 \pm 0.98^{*}$ & $18.92 \pm 0.29^{*}$ & $15.34 \pm 0.17^{*}$ \\
\hline
\end{tabular}

Table 1. Protective effect of APPI and APPII reflected in cellular indexes. Data are shown as means \pm SEM $(\mathrm{n}=3)$. $* \mathrm{P}<0.05$ versus the model group as revealed by ANOVA.

enzymes ${ }^{6}$. Thus, activities of extracellular aminotransferases can reflect the status of liver cells, with higher values reflecting liver damage. It was shown that the activities of extracellular alanine and aspartate aminotransferases (ALT and AST) of the model group were elevated relative to those of the control group, implying that pathogenetic factors of FFA and ethanol inflicted damage on the cells. However, APPI and APPII mitigated this damage, and the elevated activities of alanine and aspartate aminotransferases consequently declined $(\mathrm{P}<0.05)$, as shown in Table 1.

Mechanisms of APPI and APPII. Adiponectin is an adipocyte-derived cytokine. Adiponectin and its receptor 2 abundant in human hepatocytes, constitute a complex, and play a central role in the pathogenesis of liver disorders. Adiponectin, which has an anti-steatotic effect on liver cells, acts mainly through the AMPK and PPAR $\alpha$ pathway. It promotes oxidation of FFA and suppresses gluconeogenesis, entry of FFA and de novo lipid synthesis ${ }^{7,8}$. When drugs stimulate the adipocytes, adiponectin is secreted and conjugated with Adipor 2 receptors on the surface of the hepatocytes. The complex activates $A M P K$ gene expression, induces genes including $C P T l$ and $A C O X 1$ in FFA oxidation pathway upregulation. Meanwhile, $A M P K$ induces activation of transcription factor $P P A R \alpha$ and accelerates FFA metabolism.

In order to validate the mechanism of APPI and APPII, five key genes have been selected for qRT-PCR analysis (Fig. 3). The results showed that the qRT-PCR data of these genes were consistent with results of the bioassays. For example, Adipor 2 gene was significantly down-regulated in the model group, as well as $A M P K, C P T l, P P A R \alpha$ and $A C O X 1$, which deactivate FFA oxidation process and accelerate FFA accumulation in hepatocytes or hepatic tissue. It was disclosed that the TG concentration of the model group rose. It was found that Adipor 2 gene was up-regulated in APPI and APPII groups, the same took place regarding AMPK, CPTl, PPAR $\alpha$ and ACOX1. The results indicated that APPI and APPII could stimulate adipocytes to secrete adiponectin and activate the FFA metabolic pathway, reducing lipid accumulation. 

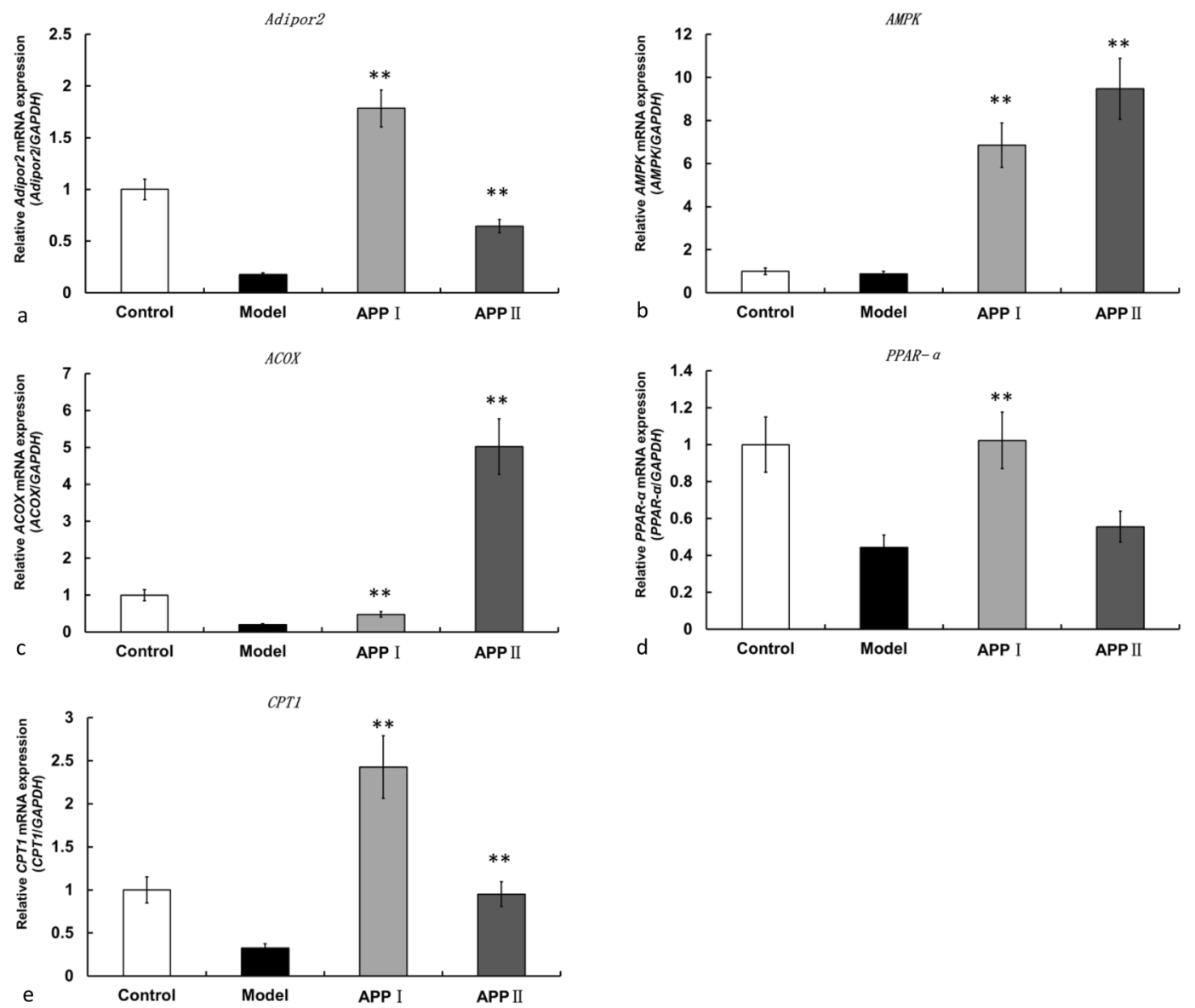

Figure 3. q-PCR analysis of APPI and APPII on expression of genes in the adiponectin pathway. Data are shown as means $\pm S E M(n=3) . * * P<0.01$ versus the model group as revealed by ANOVA.

Hepatoprotective effect of APPI and APPII in vivo. Protective effects of APPI and APPII on hepatic damage. FLD is the leading cause of chronic liver disease worldwide. Unhealthy high fat diets and sedentary habits result in the high prevalence of FLD. The rats were randomized to receive either standard laboratory diet (SLD) or high cholesterol and high fat diet (HFD) for 8 weeks. The biochemical analysis showed that HFD induced a rise in the levels of TG, total cholesterol (TC) and low-density lipoprotein cholesterol (LDL-C), and the activities of ALT and AST in serum (see Supplementary Table S1). The HFD rats gained weight faster than those with SLD, at the ratio of $105.43 \%$, and the liver weights in the model groups were heavier than that in control group. AST and ALT activities in the serum have been employed as biochemical markers for hepatic damage. The heightened AST and ALT expression levels signified the enhanced permeability and injury of hepatocytes.

In the present study, APPI and APPII ameliorated the biochemical indexes. Significant decreases $(\mathrm{P}<0.05)$ in ALT and AST activities were observed in APPI and APPII-treated groups similar to the simvastatin- treated group (Table 2). It indicated that APPI and APPII could protect hepatocytes from injury and maintain their integrity. The concentrations of TC, TG, LDL-C, and high density lipoprotein cholesterol (HDL-C) in the serum are vital parameters in the analysis of lipid metabolism. Abnormal serum lipid indicated the risks of FLD. The TC, TG and LDL-C concentrations of serum in APPI or APPII-treated groups were reduced relative to those in the model group $(\mathrm{P}<0.05)$. It was indicated that APPI and APPII might be useful for preventing FLD through improving lipid metabolism.

HFD is associated with fat accumulation in liver. As shown in Fig. 4, hepatic TG level was elevated significantly by 2.07 fold versus the control group. However, treatment of HFD with APPI and APPII, just like treatment with simvastatin, significantly $(\mathrm{P}<0.05)$ lowered the TG level in liver tissue compared to the model group.

Histopathological observations. Histopathological examination was used to evaluate the hepatoprotective effects of APPI and APPII on HFD-elicited acute liver lesion. Figure 5b revealed that the nuclei of hepatocytes in the model group were irregular, lysis diffluence and even disappearance. The cytoplasm exhibited a ballooning degeneration with fatty droplets. It was also observed that hepatic sinus appeared eclasis and gore, some of Kupffer cells proliferated. It suggested that the tissues in the model group turned to hepatocyte injury and the FLD model was successfully established. However, the hepatocytes showed a regular arrangement, the cytoplasmic ballooning degeneration was mitigated in APPI and APPII with HFD groups (Fig. $5 \mathrm{c}-\mathrm{f}$ ). In the APPI and APPII treated groups, lipid and hepatocyte injury were reduced relative to the model group. Hepatocyte volume was reduced, liver lobules were distinctly delineated, and there were fewer fat droplets. These histological alterations 


\begin{tabular}{|l|l|l|l|l|l|l|}
\hline & TG $(\mathbf{m m o l} / \mathbf{L})$ & TC $(\mathbf{m m o l} / \mathbf{L})$ & $\begin{array}{l}\text { HDL-C } \\
(\mathbf{m m o l} / \mathbf{L})\end{array}$ & $\begin{array}{l}\text { LDL-C } \\
(\mathbf{m m o l} / \mathbf{L})\end{array}$ & AST (U/L) & ALT (U/L) \\
\hline Control group(SLD) & $0.92 \pm 0.23^{*}$ & $2.36 \pm 0.28^{*}$ & $1.52 \pm 0.19^{*}$ & $0.44 \pm 0.09^{*}$ & $133.00 \pm 16.54^{*}$ & $136.60 \pm 24.31^{*}$ \\
\hline Model group(HFD) & $1.96 \pm 0.21$ & $3.98 \pm 0.44$ & $1.01 \pm 0.11$ & $2.78 \pm 0.43$ & $215.40 \pm 15.84$ & $233.00 \pm 19.72$ \\
\hline APPI + HFD group $(50 \mathrm{mg} / \mathrm{kg} / \mathrm{d})$ & $0.44 \pm 0.08^{*}$ & $2.48 \pm 0.08^{*}$ & $1.46 \pm 0.14^{*}$ & $0.66 \pm 0.13^{*}$ & $148.50 \pm 20.41^{*}$ & $160.00 \pm 14.83^{*}$ \\
\hline APPI + HFD group $(100 \mathrm{mg} / \mathrm{kg} / \mathrm{d})$ & $0.39 \pm 0.09^{*}$ & $2.20 \pm 0.3^{*}$ & $1.59 \pm 0.21^{*}$ & $0.60 \pm 0.08^{*}$ & $151.00 \pm 13.18^{*}$ & $172.33 \pm 27.26^{*}$ \\
\hline APPII + HFD group $(50 \mathrm{mg} / \mathrm{kg} / \mathrm{d})$ & $0.33 \pm 0.03^{*}$ & $2.15 \pm 0.24^{*}$ & $1.37 \pm 0.33$ & $0.57 \pm 0.06^{*}$ & $211.33 \pm 15.75$ & $215.67 \pm 12.93$ \\
\hline APPII + HFD group $(100 \mathrm{mg} / \mathrm{kg} / \mathrm{d})$ & $0.24 \pm 0.03^{*}$ & $1.73 \pm 0.33^{*}$ & $1.11 \pm 0.22$ & $0.50 \pm 0.10^{*}$ & $148.33 \pm 7.95^{*}$ & $126.83 \pm 3.99^{*}$ \\
\hline $\begin{array}{l}\text { Simvastatin } \\
(2 \mathrm{mg} / \mathrm{kg} / \mathrm{d})\end{array}$ & $0.64 \pm 0.08^{*}$ & $2.02 \pm 0.25^{*}$ & $1.19 \pm 0.17$ & $0.73 \pm 0.08^{*}$ & $109.33 \pm 26.63^{*}$ & $101.00 \pm 21.57^{*}$ \\
\hline HFD-SLD group & $0.91 \pm 0.12^{*}$ & $2.16 \pm 0.11^{*}$ & $1.34 \pm 0.08^{*}$ & $0.67 \pm 0.03^{*}$ & $115.20 \pm 14.01^{*}$ & $121.80 \pm 30.24^{*}$ \\
\hline
\end{tabular}

Table 2. Hepatoprotective effects of APPI and APPII on serum biochemical markers. Data are shown as means \pm SEM $(n=6)$. $* P<0.05$ versus the model group as revealed by ANOVA.SLD $=$ standard laboratory diet. $\mathrm{HFD}=$ high fat diet.

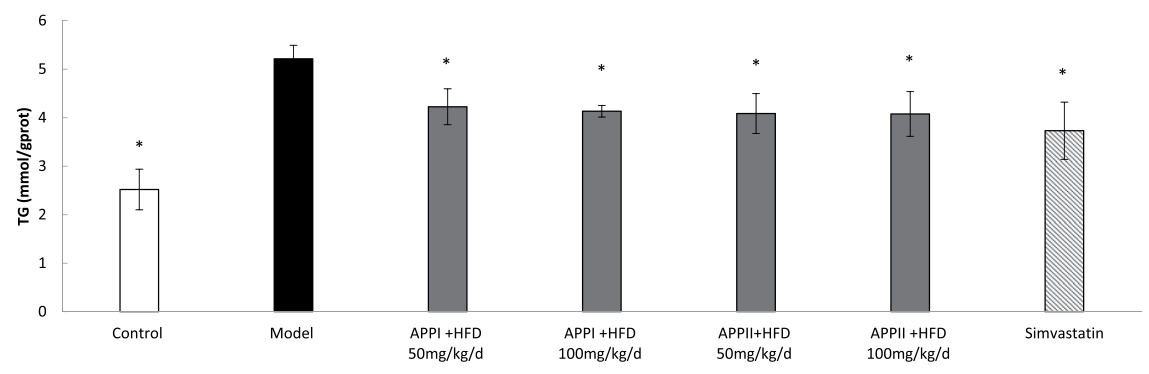

Figure 4. Hepatic TG content in rats. Data are shown as means $\pm \operatorname{SEM}(n=6) . * P<0.05$ versus the model group as revealed by ANOVA. Control rats were fed standard laboratory diet (SLD)while the rest were fed a high fat diet (HFD).

were in agreement with the serum and liver lipid profiles shown in Fig. 4 and Table 2. Therefore, APPI and APPII minimized lipid deposition in the liver cells of FLD rats.

\section{Discussion}

In our earlier study a soluble polysaccharide was prepared from A. polytricha fruiting bodies with an extraction rate approximating $20 \%$ by using the following conditions: extraction with $28 \mathrm{~mL}$ water/g mushroom at $95^{\circ} \mathrm{C}$ for $4 \mathrm{~h}$. The polysaccharide preparation exerted an antihypercholesterolemic action in diet-induced hypercholesterolemic rats and depressed the plasma cholesterol level by about one-third at the doses of $4.5 \mathrm{and} 9 \mathrm{mg} / \mathrm{kg} / \mathrm{d}$. In the present investigation the polysaccharide was fractionated into two polysaccharide-peptides APPI and APPII have similar monosaccharide moieties and FITR spectra but differ in molar ratio of the monosaccharides and molecular weight. APPII also differs from APPI in exhibiting absorption around $1420.96 \mathrm{~cm}^{-1}$ probably indicating deforming vibrations of the C-H bond and absorption at $1375.60 \mathrm{~cm}^{-1}$ and $1250.16 \mathrm{~cm}^{-1}$ signifying the presence of a carboxyl group and a sulfate radical. Although APPI and APPII possess distinct N-terminal and internal partial amino acid sequences, both of them resemble other mushrooms in these sequences. In APPI, two of the internal partial amino acid sequences closely resemble those of Cordyceps militaris mannose-binding lectin. In APPII, two of the internal partial amino acid sequences manifest pronounced similarity to those of Flammulina velutipes immunomodulatory protein and Lentinula edodes peptidyl-prolyl cis-trans isomerase, respectively.

Auricularia auricular polysaccharides simulated hydrolysates, derived from $A$. auricular which is a species very closely similar to A. polytricha, were composed of arabinose, galactose, glucosamine, glucose, xylose and mannose with the molar ratio of 1.91: 0.67:0.23:1.00: 0.52: $2.89^{59}$. A. auricular polysaccharide was composed of arabinose, galactose, glucosamine, glucose, rhamnose and mannose with the molar ratio of 0.93:0.91:4.32:1:37.53 ${ }^{60}$. APPI isolated in the present study was composed of arabinose, galactose, glucose, xylose and mannose in a molar ratio of 1:4.4:15.4:38.3:46.2, while the corresponding molar ratio for APPII was 1:71.5:99.2: 10: 5.1. Glucosamine was lacking in APPI and APPII. The data showed that they were all different in the composition of the sugar moiety.

The FT-IR spectrum of APPI exhibited a broad peak at $3400.42 \mathrm{~cm}^{-1}$ characteristic of hydroxyl groups with stretching vibration, and a peak at $2934.88 \mathrm{~cm}^{-1}$ due to $\mathrm{C}-\mathrm{H}$ stretching vibration. The absorption peak at $1250.16 \mathrm{~cm}^{-1}$ revealed that the polysaccharide-peptide APPII possessed a sulfate radical. In the study of Zhang et al. ${ }^{61}$, the FT-IR spectrum of A. auricular polysaccharide also displayed a band in the region of 3411.1 and $3410.5 \mathrm{~cm}^{-1}$ corresponding to the hydroxyl stretching vibration of the polysaccharide and at 2923.3 and $2921.3 \mathrm{~cm}^{-1}$ corresponding to a weak $\mathrm{C}-\mathrm{H}$ stretching vibration at $1258 \mathrm{~cm}^{-1}$ due to an asymmetrical S=O stretching vibration. Although there were similarities the spectra of the aforementioned polysaccharides were not identical.

With regard to its effect on lipid metabolism, an aqueous extract of A. polytricha, which was composed of phenolics, tannins and polysaccharides, was found to reduce lipid storage, inflammation, proinflammatory 


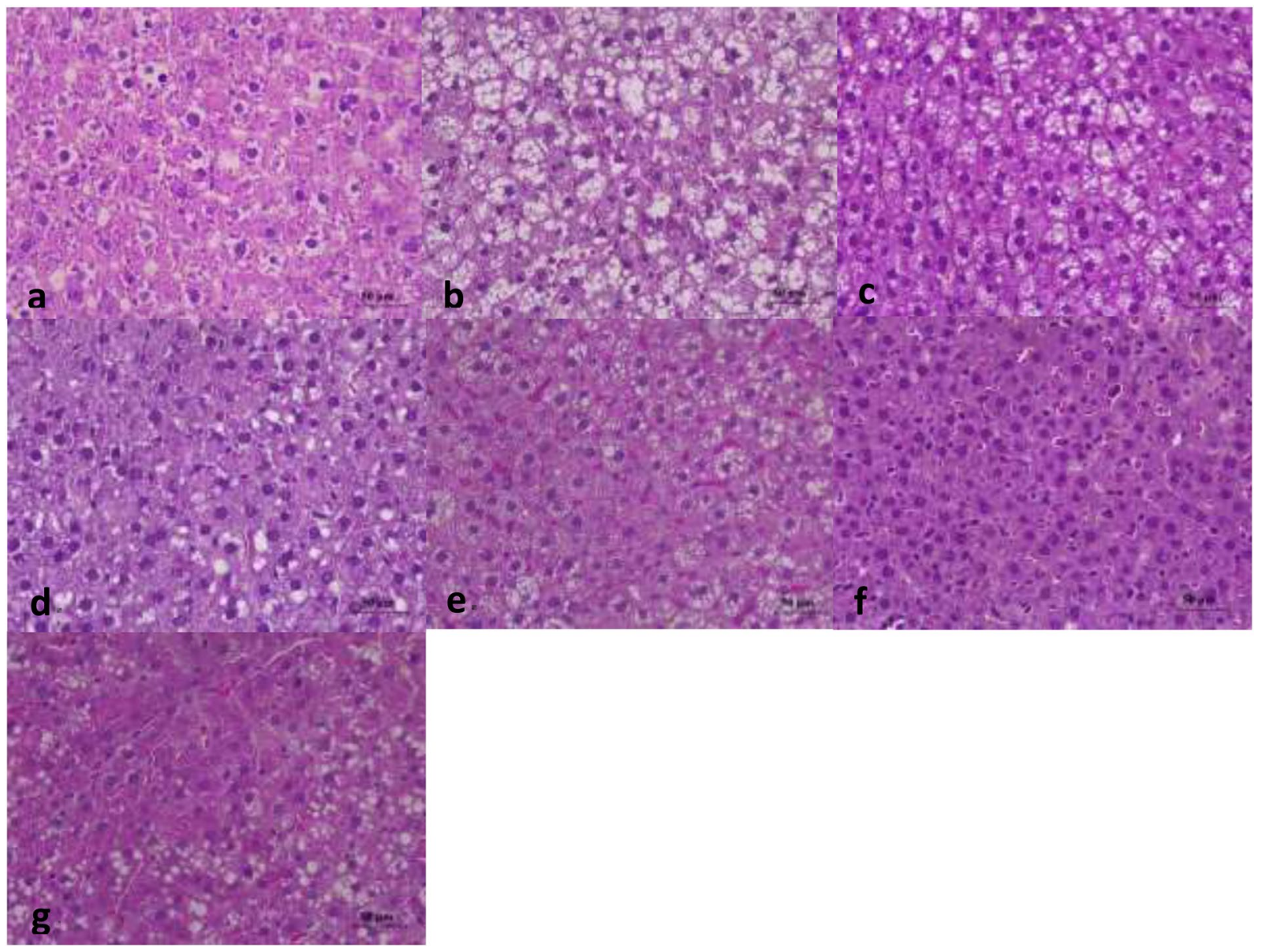

Figure 5. Histopathological alterations in rat livers, stained with $H \& E(400 \times)$. (a) Control group, (b) Model group, (c) APPI + HFD group (50 mg/kg/d), (d) APPI + HFD group (100 mg/kg/d), (e) APPII + HFD group $(50 \mathrm{mg} / \mathrm{kg} / \mathrm{d}),(\mathbf{f})$ APPII + HFD group $(100 \mathrm{mg} / \mathrm{kg} / \mathrm{d})$, (g) Simvastatin group $(2 \mathrm{mg} / \mathrm{kg} / \mathrm{d})$. Control rats were fed standard laboratory diet (SLD) while the rest were fed a high fat diet (HFD).

cytokines and oxidative stress in rats provided with a high-fat diet containing 10\% lard. Upon completion of the treatment which lasted for 12 weeks. It was observed that changes brought about by the high-fat diet including the hepatosomatic index, serum activities of aminotransferases, triglyceride, serum levels of free fatty acid, total cholesterol, high density lipoprotein cholesterol, low density lipoprotein cholesterol, very low density lipoprotein cholesterol, antioxidant vitamins $\mathrm{C}$ and $\mathrm{E}$, malondialdehyde, and the proinflammatory cytokines interleukin- 6 and tumor necrosis factor-alpha, were at least partially reversed by treatment with the A. polytricha polysaccharide-peptides ${ }^{62}$. Alterations in hepatic triglyceride, cholesterol, malondialdehyde, antioxidant vitamins $\mathrm{E}$ and $\mathrm{C}$, and antioxidant enzymes were also attenuated ${ }^{62}$. Intake of a Ganoderma lucidum preparation enriched in polysaccharide-peptides and triterpenoids by human subjects restored the hepatic condition to normal from the initially mild fatty liver as evidenced by abdominal ultrasonography and reduction in plasma activities of aminotransferases ${ }^{63}$. However, it is known that phenolics and tannins also displayed similar activities ${ }^{64}$. Thus, the aforementioned actions of the aqueous extract of A. polytrich $a^{62}$ were probably attributed to each of its constituents: phenolics, tannins and polysaccharides, and the action of the Ganoderma lucidum preparation ${ }^{63}$ was partly due to triterpenoids ${ }^{65,66}$.

In the present investigation both APPI and APPII exhibited a lipid lowering effect in vitro as well as in vivo. In the in vitro system employing ethanol and a mixture of palmitic and oleic acids to induce hepatotoxicity, the resulting elevated level of intracellular triglyceride and activity of extracellular aspartate and alanine aminotransferase were suppressed by APPI and APPII, and the decreased cellular superoxide dismutase activity was upregulated by the two polysaccharide-peptides. APPI and APPII increased the viability of the hepatocytes. It appeared that APPII was more potent in inhibiting triglyceride accumulation in the hepatocytes. However, this was not true regarding the effects on hepatocyte viability, superoxide dismutase activity, and aminotransferases released into the culture medium. APPI had a more potent effect on Adipor 2, CPTl, and PPAR $\alpha$ whereas APPI had a higher activity on $A M P K$ and $A C O X 1$.

In the in vivo system, the high fat diet brought about a rise in the circulatory titers of triglyceride, total cholesterol, low density cholesterol, aspartate aminotransferase and alanine aminotransferase but a decline in plasma high density cholesterol. APPII was more potent than APPI in its antihypercholesterolemic and antihypertriglyceridemic and LDL lowering activities. APPI was more potent than APPII in raising plasma HDL cholesterol 
activity. APPI and APPII were similar in their activity in preventing hepatic triglyceride accumulation and secretion of aspartate aminotransferase and alanine aminotransferase into the blood stream. The hepatocytes after treatment with APPI and APPII were similar in appearance under the microscope.

Polysaccharides from A. auricula very closely similar to A. polytricha also reduced the serum concentrations of total cholesterol and low-density lipoprotein cholesterol in mice receiving a diet enriched in cholesterol and improved lipoprotein lipase activity and total antioxidant capacity ${ }^{67}$. A water-soluble crude polysaccharide from A. auricular mycelia grown under solid-state fermentation lowered serum concentrations of triglyceride, total cholesterol, and low-density lipoprotein cholesterol in a high fat diet induced hyperlipidemic mice ${ }^{60}$. A. auricular polysaccharides simulated hydrolysates from the dried fruiting bodies induced a decline in the serum concentrations of triglyceride and low-density lipoprotein cholesterol without affecting high-density lipoprotein cholesterol and total cholesterol in streptozotocin -induced diabetic rats ${ }^{59}$. The alcohol extract of $A$. auricula-judae provided to mice on a high fat diet reduced plasma lipids and hepatic enzymes and downregulated expression of adipogenic/lipogenic genes (PPAR $\gamma, \mathrm{C} / \mathrm{EBP} \alpha, \mathrm{FAS})$ in $3 \mathrm{~T} 3-\mathrm{L} 1$ cells $^{68}$. An alcohol extract of $A$. auricula enriched in polyphenolics lowered serum total cholesterol and upregulated high-density lipoprotein cholesterol level and fecal bile acid excretion. However, it was devoid of any action on serum low-density lipoprotein cholesterol and triglycerides and fecal neutral cholesterol excretion ${ }^{59}$. The effects of Auricularia cornea polysaccharides and enzymatic-extractable polysaccharides displayed antioxidant and reactive oxygen species scavenging activities in vitro and exerted a protective action on alcohol-induced liver pathology in mice. The polysaccharides produced their hepatoprotective effects by suppressing lipid peroxidation, facilitating alcohol metabolism, and downregulatiing the expression of inflammatory mediators and preventing the alcohol-induced histopathological alterations ${ }^{69}$.

Adiponectin is anti-inflammatory and insulin-sensitizing adipokine. Adiponectin deficiency linked with an inflammatory state is present in obesity, severe chronic hepatitis C-related steatosis, non-alcoholic fatty liver diseases such as hepatic steatosis and non-alcoholic steatohepatitis, and cancer ${ }^{70-74}$. Adiponectin reduces insulin resistance, prevents excess hepatic lipid accumulation, inhibits inflammation and fibrosis, and exerts a hepatoprotective action ${ }^{75-77}$. It also protects against cerebrovascular, cardiovascular, and chronic renal diseases ${ }^{7,71,72,76-80}$. It is a target in the therapy of obesity, cardiovascular and inflammatory ailments, nonalcoholic steatohepatitis, diabetes and neurodegenerative diseases ${ }^{7,76,78-80}$. Efforts are made to upregulate adiponectin by therapeutic medications and/or changes in lifestyle 77 .

Mushroom extracts exhibit a stimulatory effect on adiponectin production. An elevation of the circulatory adipokine level, decline in levels of markers associated with hepatic damage, and activities of key enzymes catalyzing fatty acid biosynthesis, together with attenuation of liver enlargement and triglyceride accumulation, were observed in $d b / d b$ mice after consumption of ethanol-soluble extract of Panellus serotinus ${ }^{81,82}$. Supplementation with Agaricus blazei reduces insulin resistance and improves circulatory adiponectin level in patients with type 2 diabetes Murill ${ }^{83}$. $\beta$-glucan-rich polysaccharides derived from Pleurotus sajor-caju upregulated adiponectin expression $^{84}$. The ethyl acetate fraction of Hericium erinaceus downregulated lipopolysaccharide -elicited decline of adiponectin mRNA in 3T3-L1 fat cells in coculture with RAW264 macrophages ${ }^{85}$. An aqueous extract of Antrodia cinnamomea reversed the action of a high-fat diet on body weight gain, inflammatory cytokines and adiponectin production in mice ${ }^{86}$. Adiponectin promotes fatty acid oxidation in skeletal muscle ${ }^{79}$. The purified polysaccharide- peptides acquired from A. polytricha act via genes affect fatty acid oxidation. Peroxisome proliferator-activated receptors (PPARs) are metabolic regulators of lipid and lipoprotein levels which are divided into $\alpha, \beta / \delta$ and $\gamma$ subtypes. The PPAR- $\alpha$ agonists suppress triglyceride levels. PPAR- $\gamma$ agonists demonstrate potent hypoglycemic but weaker triglyceride lowering activity. PPAR- $\alpha / \delta$ agonists display antihyperglycemic, and triglyceride lowering activities. They are promising for the therapy of atherogenic dyslipidemias and NAFLD ${ }^{87}$. PPARs are paramount to energy homeostasis of the entire body and as fatty acids sensors in several human lipid metabolic diseases ${ }^{88}$. Extracts, polysaccharides and other compounds from mushrooms inhibit fat production by acting via PPAR $\gamma$ and/or PPAR $\alpha$. This study is the first to reveal the action of mushroom polysaccharide peptides through PPAR $\alpha$.

The present report on the hepatoprotective and fatty liver alleviating activities of purified polysaccharide-peptides acquired from A. polytricha and elucidation of the mechanism involved represents the first of its kind on purified polysaccharide-peptides. The A. polytricha polysaccharide peptides are of considerable interest and promising for development into therapeutic agents in view of the array of health-enhancing activities of adiponectin.

\section{Methods}

Materials and reagents. A. polytricha fruiting bodies were collected in Beijing, China, and were ground to produce a fine powder. Monosaccharides (D-mannose, D-glucose, D-glucuronic acid, L-rhamnose, D-xylose, D-fructose, D-galacturonic acid, D-galactose and D-arabinose), DEAE-cellulose, MTT, oleic acid and palmitate were products of Sigma-Aldrich (USA). Superdex-200 column was obtained from General Electric Company (GE, USA), and HepG2 cell line was purchased from ATCC (American Type Tissue Culture Collection). Dulbecco's modified Eagle's minimum essential medium (DMEM), fetal bovine serum (FBS), phosphate buffered saline (PBS), trypsin solution, penicillin and streptomycin were products of Invitrogen (USA). The assay kits for protein content, TG, ALT, AST, and SOD were purchased from Nanjing Jiancheng Bioengineering Institute (Nanjing, Jiangsu Province, China). The other chemicals and solvents employed were of analytical reagent grade and obtained from Sinopharm Chemical Reagent Co., Ltd. (Shanghai, China).

Extraction and isolation of polysaccharide-peptides. Crude polysaccharide-peptides were extracted from A. polytricha following published procedures ${ }^{41}$. Briefly, the dry powder was extracted thrice, for $4 \mathrm{~h}$ each time, by employing 40 volumes of hot water $\left(90^{\circ} \mathrm{C}\right)$. The extracts were combined and concentrated in a rotary 
evaporator. The concentrated solution was deproteinated using Sevag reagent (chloroform and n-butanol, 4:1 vol:vol $)^{89}$. Ethanol (100\%) was added to the deproteinated solution which was then allowed to stand at $20^{\circ} \mathrm{C}$ overnight, and the precipitated polysaccharide-peptide (APP) was obtained by centrifugation. Subsequently, a solution of the APP in distilled water was fractionated on a DEAE-cellulose column $(1 \mathrm{~cm} \times 30 \mathrm{~cm})$ equilibrated with distilled water. The column was eluted sequentially with $0,0.2$ and $1 \mathrm{~mol} / \mathrm{L} \mathrm{NaCl}$ solution successively at a flow rate of $2.0 \mathrm{~mL} / \mathrm{min}$. The unadsorbed peak D1 and adsorbed peak D2, with the carbohydrate content measured with the phenol-sulfuric acid method, were enriched in polysaccharide. The D1 and D2 fractions were collected, concentrated, and further fractionated on an FPLC-Superdex 200 10/300 column in $0.2 \mathrm{~mol} / \mathrm{L} \mathrm{NH}_{4} \mathrm{HCO}_{3}(\mathrm{pH}$ 8.5) buffer using an AKTA Purifier (GE Healthcare), respectively to obtain bioactive polysaccharide-peptides APPI and APPII.

Characterization of bioactive polysaccharide-peptides. Analysis of monosaccharide composition. Monosaccharide compositions of APPI and APPII were determined with a gas chromatography mass spectrometer (GC-MS). The sample was hydrolyzed following the method of Yu et al. ${ }^{90}$.

FT-IR analyses. The IR spectra of APPI and APPII were obtained by using a Fourier transform infrared spectrophotometer (Nicolet iS5 FTIR Spectrometer, USA) within the wave number range of 4000 to $400 \mathrm{~cm}^{-1}$. The dried sample was ground with spectroscopic grade potassium bromide $(\mathrm{KBr})$ powder and then pressed into 1- $\mathrm{mm}$ pellets.

Determination of molecular weight. The purity and molecular weights of APPI and APPII were assessed with HPGPC technique using HPLC on a TSK GMPWXL column. The samples were injected and eluted at $0.6 \mathrm{~mL} /$ min with $0.1 \mathrm{~mol} / \mathrm{L} \mathrm{NaNO}_{3}$ and $0.05 \% \mathrm{NaN}_{3}$ as the mobile phase.

$N$-terminal and inner amino acid sequence analysis. The polysaccharide-peptides band excised from the SDS-PAGE gel was transferred to a polyvinylidenedifluoride (PVDF) membrane by Western blotting and then stained with Coomassie brilliant blue R-250. Analysis of the stained band was performed with the automated Edman degradation method ${ }^{91}$.

The polysaccharide-band of SDS-PAGE gel was recovered and dispatched to Tsinghua University (Beijing, China) for partial amino acid sequence analysis. Sequence homology with known sequences was searched using the BLAST/NCBI database.

Assay for the protective effects of APPI and APPII on hepatocytes. Preparation of model of hepatocyte injury-induced by ethanol and a mixture of palmitic and oleic acids. FFA and ethanol were considered as the pathogenetic factors which induce hepatocyte injury. FFA used was a mixture of palmitic and oleic acids, prepared according to the method described by Garcia et al. ${ }^{92}$. The hepatocyte injury model was established as follows. HepG2 (liver cancer) cells were cultured in Dulbecco's Modified eagle medium (DMEM) medium containing $10 \%(\mathrm{v} / \mathrm{v})$ fetal bovine serum (FBS), $100 \mathrm{IU} / \mathrm{mL}$ penicillin and $100 \mathrm{mg} / \mathrm{L}$ streptomycin, at $37^{\circ} \mathrm{C}$ in a humidified atmosphere of $5 \%(\mathrm{v} / \mathrm{v}) \mathrm{CO}_{2}$. Cells were then seeded onto 96 -well plates at a concentration of $8 \times 10^{3}$ cells/well, and allowed to incubate for $12 \mathrm{~h}$ before addition of FFA and ethanol. Incubation was then conducted for $24 \mathrm{~h}$. Afterwards, the MTT assay was performed to measure cell viability. As a control, PBS instead of pathogenetic factors (FFA and ethanol) was added to the wells.

Protective effects and mechanisms of APPI and APPII on hepatocytes. To determine hepatoprotective effects, injured HepG2 cells were treated with APPI and APPII at various concentrations for $48 \mathrm{~h}$. Upon termination of the incubation, viability of the cells was measured with the MTT assay. The optimal doses, obtained by using cell viability determination, were considered as effective concentrations to analyze the mechanism of APPI and APPII. HepG2 cells were seeded onto 6-well plates at a concentration of $3 \times 10^{4}$ cells/well, for preparation of the injury models before addition of APPI and APPII at optimal doses. Following incubation for $48 \mathrm{~h}$, the cells and culture medium in all treatment groups were collected to evaluate the repairing ability. Intracellular protein, cellular TG and the activities of SOD from cells were quantified using commercial assay kits, and the activities of ALT and AST in the culture medium were assayed with colorimetric assay kits, all in accordance with the manufacturers' protocols. Histological analysis of cellular lipid was determined with the Oil-red O stain method.

Gene expression analysis. To analyze the therapeutic pathways of APPI and APPII, the expression levels of lipid metabolism genes in the adiponectin pathway were determined by qRT-PCR. Extraction of total RNA from the samples was conducted by using TRIzol reagent (Invitrogen, USA) and reversely transcribed with oligo (dT) using EasyScriptTM First-Strand cDNA Synthesis SuperMix (Transgen, China), in accordance with the manufacturer's protocol. qRT-PCR was performed following the protocol of the Maxima SYBR Green/ROX qRT-PCR Master Mix (Fermentas, USA) employing an ABI 7500 (Applied Biosystems, USA). The GAPDH RNA level was used as an endogenous control for mRNAs. The qRT-PCR procedure comprised pre-denaturation at $95^{\circ} \mathrm{C}$ for $5 \mathrm{~min}$ and 40 cycles which consisted of exposure to $95^{\circ} \mathrm{C}$ for 30 seconds, and to $60^{\circ} \mathrm{C}$ for $1 \mathrm{~min}$. The relative expression level was computed by the 2 - $\ddot{A} \mathrm{ACt}$ method. Five independent experiments were performed.

Protective effects of APPI and APPII against fat-induced fatty liver in rats. Experimental animals and diets. Male Wistar rats (220-280 g) obtained from the Institute of Laboratory Animal Science, Chinese Academy of Medical Sciences, were utilized in this study. All experimental protocols were approved by the University Safety Office and Animal Experimentation Ethics Committee at The Chinese University of Hong Kong and China Agricultural University. All animal experiments were carried out in accordance with the 
approved guidelines of the Animal Care and Use Committee of The Chinese University of Hong Kong and China Agricultural University. The normal control group was fed with SLD, while the other groups received a HFD including $2 \%$ cholesterol and $25 \%$ pig fat.

Animal grouping and experimental design. Rats were randomly allocated in seven groups with six rats in a group. The normal control group was fed SLD, while the other six groups received a HFD including $2 \%$ cholesterol and 25\% pig fat for eight weeks. The six HFD groups were treated as follows: (i) the model group (no treatment), (ii) low-dose APPI group (50 mg/kg/d), (iii) high-dose APPI group (100 mg/kg/d), (iv) low-dose APPII group (50 mg/kg/d), (v) high-dose APPII group (100 mg/kg/d), (vi) positive group of simvastatin treatment $(2 \mathrm{mg} / \mathrm{kg} / \mathrm{d})$. APPI, APPII and simvastatin were administered intragastrically for 4 weeks after an 8-weeks HFD model foundation. Rats in the normal control group and model control group an equal volume of normal saline instead. Free access to food and water was allowed. The rats were weighed weekly, and fresh food was provided daily.

After the final drug treatment, the animals were sacrificed by cervical dislocation, and blood was collected for biochemical analysis. The liver was removed and weighed. A small piece of the liver was fixed in $10 \%$ buffered formalin solution for histological processing, and the remainder was frozen at $-80^{\circ} \mathrm{C}$ till biochemical analysis.

Histological analysis and biochemical evaluation. The hepatic tissues were preserved in $4 \%$ paraformaldehyde at $4{ }^{\circ} \mathrm{C}$ for $24 \mathrm{~h}$ before embedding in paraffin for sectioning. The tissue sections were prepared and stained with hematoxylin and eosin (H\&E). Hepatic histology was observed using an Olympus microscope, IX71 (Olympus, Japan).

Liver tissue was homogenized using phosphate buffer saline. Following centrifugation at 10,000 r/min for $40 \mathrm{~min}$ at $4{ }^{\circ} \mathrm{C}$, the supernatant was saved for biochemical evaluation. TC and TG levels as lipid indexes, and ALT and AST activities as hepatic injury indexes were assayed using commercial kits following the manufacturer's instructions.

Statistical analysis. All experiments in all of the bioassays were conducted in triplicate. Data are shown as mean \pm standard deviation (SD). Statistical analysis was conducted with one-way analysis of variance (ANOVA) using SPSS software (SPSS 16.0 software package, USA). Data were assessed by using ANOVA and $\mathrm{P} \leq 0.05$ was considered to be statistically significant.

\section{Conclusion}

The present report on the hepatoprotective and fatty liver alleviating activities of purified polysaccharide-peptides acquired from $A$. polytricha and elucidation of the mechanism involved represents the first of its kind on purified polysaccharide-peptides. The A. polytricha polysaccharide peptides are of considerable interest and promising for development into a therapeutic agent in view of the array of health-enhancing activities of adiponectin.

\section{References}

1. Yang, Z. et al. Protective effect of Anoectochilus roxburghii polysaccharide against CCl4-induced oxidative liver damage in mice. Int. J. Biol. 96, 442-450 (2017).

2. Wang, X. Y., Luo, J. P., Chen, R., Zha, X. Q. \& Pan, L. H. Dendrobium huoshanense polysaccharide prevents ethanol-induced liver injury in mice by metabolomic analysis. Int. J. Biol. 78, 354-362 (2015).

3. Yin, Y. et al. Luteolin improves non-alcoholic fatty liver disease in $d b / d b$ mice by inhibition of liver X receptor activation to downregulate expression of sterol regulatory element binding protein 1c. Biochem. Biophys. Res. Commun. 482, 720-726 (2017).

4. Chalasani, N. et al. The diagnosis and management of non-alcoholic fatty liver disease: Practice guideline by the American gastroenterological association, american association for the study of liver diseases, and American College of Gastroenterology. Gastroenterology 142, 1592-1609 (2012).

5. Doulberis, M., Kotronis, G., Gialamprinou, D., Kountouras, J. \& Katsinelos, P. Non-alcoholic fatty liver disease: An update with special focus on the role of gut microbiota. Metabolism 71, 182-197 (2017).

6. Kew, M. C. Serum aminotransferase concentration as evidence of hepatocellular damage. The Lancet 355, 591-592 (2000).

7. Polyzos, S. A., Kountouras, J. \& Mantzoros, C. S. Adipokines in nonalcoholic fatty liver disease. Metabolism 65, 1062-1079 (2016).

8. Geng, T. et al. Identification of protective components that prevent the exacerbation of goose fatty liver: Characterization, expression and regulation of adiponectin receptors. Comp. Biochem. Physiol. B: Biochem. Mol. Biol. 194-195, 32-38 (2016).

9. Scott, E. \& Anstee, Q. Genetics of alcoholic liver disease and non-alcoholic steatohepatitis. Clin. Med. (Lond) 18, s54-s59 (2018).

10. Schild, M. \& Guy, C. Nonalcoholic steatohepatitis: histopathology basics within a broader context. Surg. Pathol. Clin. 11, 267-285 (2018).

11. Dowla, S., Aslibekyan, S., Goss, A., Fontaine, K. \& Ashraf, A. Dyslipidemia is associated with pediatric nonalcoholic fatty liver disease. J. Clin. Lipidol. (2018)

12. Kozarski, M. Dietary polysaccharide extracts of Agaricus brasiliensis fruiting bodies: chemical characterization and bioactivities at different levels of purification. Food Res. Int 64, 53-64 (2014).

13. Xin, T. et al. Extraction, purification and antitumor activity of a water-soluble polysaccharide from the roots of Polygala tenuifolia. Carbohydr. Polym. 90, 1127-1131 (2012).

14. Li, L. et al. The renal protective effects of Anoectochilus roxburghii polysaccharose on diabetic mice induced by high-fat diet and streptozotocin. J. Ethnopharmacol 178, 58-65 (2016).

15. Zhang, J. G., Liu, Q., Liu, Z. L., Li, L. \& Yi, L. T. Antihyperglycemic activity of Anoectochilus roxburghii polysaccharose in diabetic mice induced by high-fat diet and streptozotocin. J. Ethnopharmacolo 164, 180-185 (2015).

16. Chiu, C. H. et al. Polysaccharide extract of Cordyceps sobolifera attenuates renal injury in endotoxemic rats. Food Chem. Toxicol. 69, 281-288 (2014)

17. Li, Z. et al. Protective effect of a polysaccharide from stem of Codonopsis pilosula against renal ischemia/reperfusion injury in rats. Carbohydr. Polym. 90, 1739-1743 (2012).

18. Li, S. et al. Purification, antitumor and immunomodulatory activity of polysaccharides from soybean residue fermented with Morchella esculenta. Int. J. Biol. Macromol. 96, 26-34 (2017).

19. Zeng, B. et al. Protective effect of a polysaccharide from Anoectochilus roxburghii against carbon tetrachloride-induced acute liver injury in mice. J. Ethnopharmacol 200, 124-135 (2017). 
20. Yan, Z. et al. Protective effects of Ginkgo biloba leaf polysaccharide on nonalcoholic fatty liver disease and its mechanisms. Int. J. Biol. Macromol. 80, 573-580 (2015).

21. Fan, S. et al. Protective properties of combined fungal polysaccharides from Cordyceps sinensis and Ganoderma atrum on colon immune dysfunction. Int. J. Biol. Macromol. 114, 1049-1055 (2018).

22. Liao, B. \& Huang, H. Structural characterization of a novel polysaccharide from Hericium erinaceus and its protective effects against $\mathrm{H}_{2} \mathrm{O}_{2}$-induced injury in human gastric epithelium cells. J. Funct. Foods. 56, 265-275 (2019).

23. Liu, Y., Zheng, C., Su, L., Wang, Q. \& Li, Y. Protective effect of polysaccharide from Agaricus bisporus in Tibet area of China against tetrachloride-induced acute liver injury in mice. Int. J. Biol. Macromol. 118, 1488-1493 (2018).

24. Soares, A. et al. Hepatoprotective effects of mushrooms. Molecules 18, 7609-7630 (2013).

25. Zhang, J. et al. Bioactivities and health benefits of mushrooms mainly from China. Molecules 21, 938 (2016).

26. Wasser, S. P. Medicinal mushrooms in human clinical studies. Part I. anticancer, oncoimmunological, and immunomodulatory activities: A review. Int. J. Med. Mushrooms 19, 279-317 (2017).

27. Gan, D., Ma, L., Jiang, C., Wang, M. \& Zeng, X. Medium optimization and potential hepatoprotective effect of mycelial polysaccharides from Pholiota dinghuensis Bi against carbon tetrachloride-induced acute liver injury in mice. Food Chem. Toxicol. 50, 2681-2688 (2012).

28. Huang, J. et al. Hepatoprotective effects of polysaccharide isolated from Agaricus bisporus industrial wastewater against $\mathrm{CCl}_{4^{-}}$ induced hepatic injury in mice. Int. J. Biol. Macromol. 82, 678-686 (2016).

29. Liu, M., Meng, G., Zhang, J., Zhao, H. \& Jia, L. Antioxidant and hepatoprotective activities of mycelia selenium polysaccharide by Hypsizigus marmoreus SK-02. Biol. Trace. Elem. Res. 172, 437-448 (2016).

30. Liu, Q. et al. Characterization of polysaccharides with antioxidant and hepatoprotective activities from the wild edible mushroom Russula vinosa Lindblad. J. Agric. Food Chem. 62, 8858-8866 (2014).

31. Liu, Q., Zhu, M., Geng, X., Wang, H. \& Ng, T. Characterization of polysaccharides with antioxidant and hepatoprotective activities from the edible mushroom Oudemansiella radicata. Molecules 22 (2017).

32. Ozalp, F. et al. Consumption of Coprinus comatus polysaccharide extract causes recovery of alcoholic liver damage in rats. Pharm. Biol. 52, 994-1002 (2014)

33. Xu, N. et al. Hepatoprotection of enzymatic-extractable mycelia zinc polysaccharides by Pleurotus eryngii var. tuoliensis. Carbohydr. Polym. 157, 196-206 (2017)

34. Xu, N., Ren, Z., Zhang, J., Song, X. \& Jia, L. Antioxidant and anti-hyperlipidemic effects of mycelia zinc polysaccharides by Pleurotus eryngii var. tuoliensis. Int. J. Biol. Macromol. 95, 204-214 (2017).

35. Zhang, C. et al. Antihyperlipidaemic and hepatoprotective activities of acidic and enzymatic hydrolysis exopolysaccharides from Pleurotus eryngii SI-04. BMC Complement. Altern. Med. 17, 403 (2017).

36. Zhang, J. et al. Purification, characterization and hepatoprotective activities of mycelia zinc polysaccharides by Pleurotus djamor. Carbohydr. Polym. 136, 588-597 (2016).

37. Zhang, C. et al. Antioxidant and hepatoprotective activities of intracellular polysaccharide from Pleurotus eryngii SI-04. Int. J. Biol. Macromol. 91, 568-577 (2016).

38. Zhao, H. et al. The antihyperlipidemic activities of enzymatic and acidic intracellular polysaccharides by Termitomyces albuminosus. Carbohydr. Polym. 151, 1227-1234 (2016).

39. Zheng, L. et al. Antihyperlipidemic and hepatoprotective activities of mycelia zinc polysaccharide from Pholiota nameko SW-02. Int. J. Biol. Macromol. 70, 523-529 (2014).

40. Song, G. \& Du, Q. Structure characterization and antitumor activity of an $\alpha \beta$-glucan polysaccharide from Auricularia polytricha. Food Res. Int. 45, 381-387 (2012).

41. Zhao, S. et al. Extraction of a soluble polysaccharide from Auricularia polytricha and evaluation of its anti-hypercholesterolemic effect in rats. Carbohydr. Polym. 122, 39-45 (2015).

42. Park, K., Kwon, K. \& Lee, S. Evaluation of the antioxidant activities and tyrosinase inhibitory property from mycelium culture extracts. Evid. Based. Complement. Alternat. Med. 2015, 616298 (2015).

43. Chellappan, D. et al. The protective action of the aqueous extract of Auricularia polytricha in paracetamol induced hepatotoxicity in rats. Recent. Pat. Drug. Deliv. Formul. 10, 72-76 (2016).

44. Arora, S., Tandon, C. \& Tandon, S. Evaluation of the cytotoxic effects of CAM therapies: an in vitro study in normal kidney cell lines. Sci. World J. 2014, $452892(2014)$.

45. Arora, S., Goyal, S., Balani, J. \& Tandon, S. Enhanced antiproliferative effects of aqueous extracts of some medicinal mushrooms on colon cancer cells. Int. J. Med. Mushrooms 15, 301-314 (2013).

46. Song, G. \& Du, Q. Isolation of a polysaccharide with anticancer activity from Auricularia polytricha using high-speed countercurrent chromatography with an aqueous two-phase system. J. Chromatogr. A 1217, 5930-5934 (2010).

47. Zhou, J. et al. Structure analysis and antimutagenic activity of a novel salt-soluble polysaccharide from Auricularia polytricha. J. Sci. Food Agric. 93, 3225-3230 (2013).

48. Yu, J., Sun, R., Zhao, Z. \& Wang, Y. Auricularia polytricha polysaccharides induce cell cycle arrest and apoptosis in human lung cancer A549 cells. Int J biol macromol 68, 67-71 (2014)

49. Wu, N., Chiou, F., Weng, Y., Yu, Z. \& Wang, B. In vitro hypoglycemic effects of hot water extract from Auricularia polytricha (wood ear mushroom). Int. J. Food. Sci. Nutr. 65, 502-506 (2014).

50. Agarwal, K., Russo, F. \& Parks, R. Inhibition of human and rat platelet aggregation by extracts of Mo-er (Auricularia auricula). Thromb. Haemost. 48, 162-165 (1982).

51. Hokama, Y. \& Hokama, J. In vitro inhibition of platelet aggregation with low dalton compounds from aqueous dialysates of edible fungi. Res. Commun. Chem. Pathol. Pharmacol. 31, 177-180 (1981).

52. Bennett, L., Sheean, P., Zabaras, D. \& Head, R. Heat-stable components of wood ear mushroom, Auricularia polytricha (higher Basidiomycetes), inhibit in vitro activity of beta secretase (BACE1). Int. J. Med. Mushrooms 15, 233-249 (2013)

53. Liu, D. et al. Purification, characterization, and bioactivities of a polysaccharide from mycelial fermentation of Bjerkandera fumosa. Carbohydr. Polym. 167, 115-122 (2017).

54. Zhan, R., Xia, L., Shao, J., Wang, C. \& Chen, D. Polysaccharide isolated from Chinese jujube fruit (Zizyphus jujuba cv. Junzao) exerts anti-inflammatory effects through MAPK signaling. J. Funct. Foods 40, 461-470 (2018).

55. Liu, Q. et al. Purification and analysis of the composition and antioxidant activity of polysaccharides from Helicteres angustifolia L. Int. J. Biol. Macromol 30, 1263-1274 (2017).

56. Lefsih, K. et al. Pectin from Opuntia ficus indica: Optimization of microwave-assisted extraction and preliminary characterization. Food Chem. 221, 91-99 (2017).

57. Meng, M., Cheng, D., Han, L., Chen, Y. \& Wang, C. Isolation, purification, structural analysis and immunostimulatory activity of water-soluble polysaccharides from Grifola frondosa fruiting body. Carbohydr. Polym. 157, 1134-1143 (2017).

58. Fan, J. et al. Characterization, antioxidant and hepatoprotective activities of polysaccharides from Ilex latifolia Thunb. Carbohydr. Polym. 101, 990-997 (2014).

59. Lu, A. et al. Preparation of the Auricularia auricular polysaccharides simulated hydrolysates and their hypoglycaemic effect. Int. J. Biol. Macromol 106, 1139-1145 (2018).

60. Zeng, F. et al. Chemical properties of a polysaccharide purified from solid-state fermentation of Auricularia auricular and its biological activity as a hypolipidemic agent. J Food Sci 78(9), 1470-1475 (2013). 
61. Zhang, H. et al. In vitro antioxidant activities of sulfated derivatives of polysaccharides extracted from Auricularia auricular. Int $J$ Mol Sci, 2011 12(5), 3288-3302 (2011).

62. Chiu, W., Yang, H., Chiang, S., Chou, Y. \& Yang, H. Auricularia polytricha aqueous extract supplementation decreases hepatic lipid accumulation and improves antioxidative status in animal model of nonalcoholic fatty liver. Biomedicine (Taipei) 4, 12 (2014).

63. Chiu, H. F. et al. Triterpenoids and polysaccharide peptides-enriched Ganoderma lucidum: a randomized, double-blind placebocontrolled crossover study of its antioxidation and hepatoprotective efficacy in healthy volunteers. Pharm. Biol. 55, 1041-1046 (2017).

64. Zou, B. et al. Persimmon tannin accounts for hypolipidemic effects of persimmon through activating of AMPK and suppressing NF- $\kappa B$ activation and inflammatory responses in high-fat diet rats. Food Funct 5, 1536-1546 (2014).

65. Eu, C. H. A., Lim, W. Y. A., Ton, S. H. \& Kadir, K. B. A. Glycyrrhizic acid improved lipoprotein lipase expression, insulin sensitivity, serum lipid and lipid deposition in high-fat diet-induced obese rats. Lipids. Health Dis. 9, 81 (2010).

66. Chin, M. et al. Bardoxolone methyl analogs RTA 405 and dh 404 are well tolerated and exhibit efficacy in rodent models of Type 2 diabetes and obesity. Am. J. Physiol. Renal Physiol. 304, F1438-1446 (2013).

67. Chen, G. et al. Effect of polysaccharide from Auricularia auricula on blood lipid metabolism and lipoprotein lipase activity of ICR mice fed a cholesterol-enriched diet. J Food Sci 73(6), 103-108 (2008).

68. Reza, M. A. Hypolipidemic and hepatic steatosis preventing activities of the wood ear medicinal mushroom Auricularia auriculajudae (higher basidiomycetes) ethanolextract in vivo and in vitro. Int J Med Mushrooms 17(8), 723-734 (2015).

69. Wang, X. et al. Hepatoprotective effects of Auricularia cornea var. Li. polysaccharides against the alcoholic liver diseases through different metabolic pathways. Sci Rep-UK, 8(1), https://doi.org/10.1038/s41598-018-25830-w (2018).

70. Gatselis, N., Ntaios, G., Makaritsis, K. \& Dalekos, G. Adiponectin: a key playmaker adipocytokine in non-alcoholic fatty liver disease. Clin. Exp. Med. 14, 121-131 (2014).

71. Kishida, K., Funahashi, T. \& Shimomura, I. Adiponectin as a routine clinical biomarker. Best Pract. Res. Clin. Endocrinol. Metab. 28, $119-130$ (2014).

72. Katsiki, N., Mantzoros, C. \& Mikhailidis, D. Adiponectin, lipids and atherosclerosis. Curr. Opin. Lipidol. 28, 347-354 (2017).

73. Liu, D., Li, S. \& Li, Z. Adiponectin: A biomarker for chronic hepatitis C? Cytokine 89, 27-33 (2017).

74. Boutari, C., Perakakis, N. \& Mantzoros, C. Association of adipokines with development and progression of nonalcoholic fatty liver disease. Endocrinol. Metab. (Seoul) 33, 33-43 (2018).

75. Ohashi, K., Yuasa, D., Shibata, R., Murohara, T. \& Ouchi, N. Adiponectin as a target in obesity-related inflammatory state. Endocr. Metab. Immune. Disord. Drug. Targets 15, 145-150 (2015).

76. Padmalayam, I. \& Suto, M. Role of adiponectin in the metabolic syndrome: current perspectives on its modulation as a treatment strategy. Curr. Pharm. Des. 19, 5755-5763 (2013).

77. Finelli, C. \& Tarantino, G. What is the role of adiponectin in obesity related non-alcoholic fatty liver disease? World J. Gastroenter 19, 802-812 (2013).

78. Xu, Z. et al. Adiponectin attenuates streptozotocin-induced tau hyperphosphorylation and cognitive deficits by rescuing PI3K/Akt/ GSK-3 3 pathway. Neurochem. Res. 43, 316-323 (2018).

79. Ghoshal, K. \& Bhattacharyya, M. Adiponectin: Probe of the molecular paradigm associating diabetes and obesity. World J. Diabetes. 6,151-166 (2015).

80. Wang, Z. V. \& Scherer, P. E. Adiponectin, the past two decades. Journal of Molecular Cell Biology 8, 93-100 (2016).

81. Nagao, K. et al. Mukitake mushroom (Panellus serotinus) alleviates nonalcoholic fatty liver disease through the suppression of monocyte chemoattractant protein 1 production in db/db mice. J. Nutr. Biochem. 21, 418-423 (2010).

82. Inafuku, M. et al. Protective effects of fractional extracts from Panellus serotinus on non-alcoholic fatty liver disease in obese, diabetic $d b / d b$ mice. Br. J. Nutr. 107, 639-646 (2012).

83. Hsu, C. H., Liao, Y. L., Lin, S. C., Hwang, K. C. \& Chou, P. The mushroom Agaricus blazei Murill in combination with metformin and gliclazide improves insulin resistance in type 2 diabetes: A randomized, double-blinded, and placebo-controlled clinical trial. Journal of Alternative and Complementary Medicine 13, 97-102 (2007).

84. Kanagasabapathy, G., Chua, K., Malek, S., Vikineswary, S. \& Kuppusamy, U. AMP-activated protein kinase mediates insulin-like and lipo-mobilising effects of $\beta$-glucan-rich polysaccharides isolated from Pleurotus sajor-caju (Fr.), Singer mushroom, in 3T3-L1 cells. Food Chem. 145, 198-204 (2014).

85. Mori, K., Ouchi, K. \& Hirasawa, N. The anti-inflammatory effects of lion's mane culinary-medicinal mushroom, Hericium erinaceus (higher Basidiomycetes) in a coculture system of 3T3-L1 adipocytes and RAW264 macrophages. Int. J. Med. Mushrooms 17, 609-618 (2015).

86. Chang, C. et al. Antrodia cinnamomea reduces obesity and modulates the gut microbiota in high-fat diet-fed mice. Int. J. Obes. (Lond) 42, 231-243 (2018).

87. Botta, M. et al. PPAR Agonists and Metabolic Syndrome: An established role? Int. J. Mol. Sci. 19(4), https://doi.org/10.3390/ ijms19041197. (2018).

88. Xu, P., Zhai, Y. \& Wang, J. The role of PPAR and its cross-talk with CAR and LXR in obesity and atherosclerosis. Int. J. Mol. Sci. 19 (4), https://doi.org/10.3390/ijms19041260 (2018).

89. Wang, L. et al. Purification, characterization and bioactivity determination of a novel polysaccharide from pumpkin (Cucurbita moschata) seeds. Food Hydrocolloids 66, 357-364 (2017).

90. Yu, Z. et al. Characterization and biological activities of a novel polysaccharide isolated from raspberry (Rubus idaeus L.) fruits. Carbohydr. Polym. 132, 180-186 (2015).

91. Wang, S. N. et al. An extracellular yellow laccase from white rot fungus Trametes sp.F1635 and its mediator systems for dye decolorization. Biochimie 148, 46-54 (2018).

92. Garcia, M. C., Amankwa-Sakyi, M. \& Flynn, T. J. Cellular glutathione in fatty liver in vitro models. Toxicol. in Vitro 25, 1501-1506 (2011).

\section{Acknowledgements}

We are grateful to grant from Collaborative Innovation Center of Beijing Academy of Agricultural and Forestry Sciences (KJCX201915) and Beijing Academy of Agriculture and Forestry Science (KJCX20170205).

\section{Author Contributions}

S.Z. designed the purification and bioactivities studies. S.Z., Y.L. and H.W. conducted the purification and characterization studies. S.Z., S.Z., W.Z. and Y.G. conducted the bioactivities studies in vivo and in vitro. C.R. conducted the mechanistic studies. S.Z., H.W., J.H.W. and T.B. Ng were in charge of the writing of the manuscript. All authors edited the manuscript and provided comments.

Additional Information

Supplementary information accompanies this paper at https://doi.org/10.1038/s41598-019-49925-0. 
Competing Interests: The authors declare no competing interests.

Publisher's note Springer Nature remains neutral with regard to jurisdictional claims in published maps and institutional affiliations.

(c) (i) Open Access This article is licensed under a Creative Commons Attribution 4.0 International License, which permits use, sharing, adaptation, distribution and reproduction in any medium or format, as long as you give appropriate credit to the original author(s) and the source, provide a link to the Creative Commons license, and indicate if changes were made. The images or other third party material in this article are included in the article's Creative Commons license, unless indicated otherwise in a credit line to the material. If material is not included in the article's Creative Commons license and your intended use is not permitted by statutory regulation or exceeds the permitted use, you will need to obtain permission directly from the copyright holder. To view a copy of this license, visit http://creativecommons.org/licenses/by/4.0/.

(C) The Author(s) 2019 